\title{
A faint outburst of the accreting millisecond X-ray pulsar SAX J1748.9-2021 in NGC 6440
}

\author{
Pintore F. ${ }^{1 \star}$, Sanna A. ${ }^{2}$, Riggio A. ${ }^{2}$, Di Salvo T. ${ }^{3}$, Mereghetti S. ${ }^{1}$, Bozzo E. ${ }^{4}$, \\ Sánchez-Fernández C. ${ }^{5}$, Burderi L. ${ }^{2}$, Iaria R. ${ }^{3}$ \\ ${ }^{1}$ INAF-Istituto di Astrofisica Spaziale e Fisica Cosmica - Milano, via E. Bassini 15, I-20133, Milano, Italy \\ ${ }^{2}$ Università degli Studi di Cagliari, Dipartimento di Fisica, SP Monserrato-Sestu, KM 0.7, I-09042, Monserrato, Italy \\ ${ }^{3}$ Dipartimento di Fisica e Chimica, Università di Palermo, via Archirafi 36, I-90123, Palermo, Italy \\ ${ }^{4}$ ISDC, Department of Astronomy, University of Geneva, Chemin dÉcogia 16, 1290, Versoix, Switzerland \\ ${ }^{5}$ European Space Astronomy Centre (ESA/ESAC), Science Operations Department, 28691, Villanueva de la Cañada, Madrid, Spain
}

9 October 2018

\begin{abstract}
SAX J1748.9-2021 is an accreting X-ray millisecond pulsar observed in outburst five times since its discovery in 1998. In early October 2017, the source started its sixth outburst, which lasted only $\sim 13$ days, significantly shorter than the typical 30 days duration of the previous outbursts. It reached a $0.3-70 \mathrm{keV}$ unabsorbed peak luminosity of $\sim 3 \times 10^{36} \mathrm{erg} \mathrm{s}^{-1}$. This is the weakest outburst ever reported for this source to date. We analyzed almost simultaneous XMM-Newton, NUSTAR and INTEGRAL observations taken during the decaying phase of its 2017 outburst. We found that the spectral properties of SAX J1748.9-2021 are consistent with an absorbed Comptonization plus a blackbody component. The former, characterized by an electron temperature of $\sim 20 \mathrm{keV}$, a photon index of $\sim 1.6-1.7 \mathrm{keV}$ and seed photon temperature of $0.44 \mathrm{keV}$, can be associated to a hot corona or the accretion column, while the latter is more likely originating from the neutron star surface $\left(\mathrm{kT}_{b b} \sim 0.6 \mathrm{keV}, \mathrm{R}_{b b} \sim 2.5\right.$ $\mathrm{km})$. These findings suggest that SAX J1748.9-2021 was observed in a hard spectral state, as it is typically the case for accreting millisecond pulsars in outburst.
\end{abstract}

Key words: accretion, accretion discs - X-rays: binaries - X-Rays: galaxies - X-rays: individuals: SAX J1748.9-2021

\section{INTRODUCTION}

Accreting neutron stars (NS) with spin pulsations of the order of the millisecond $(\sim 1.6-10 \mathrm{~ms})$ and hosted in low mass X-ray binary systems (LMXBs) belong to the class of the accreting X-ray millisecond pulsars (AMXPs), that counts 21 sources up to date (e.g. Patruno et al. 2017; Sanna et al. 2018; Strohmayer et al. 2018). It has been proven that AMXPs are formed through the so-called recycling scenario (see e.g. Archibald et al. 2009, Papitto et al. $2013 \mathrm{~b}$, for the observational evidences of such process). According to this scenario, the period of old pulsars in binary systems can be re-accelerated to a few milliseconds from the torque exerted by matter in the accretion disc formed during the outbursts. The properties of AMXPs suggest that NS magnetic fields of the order of $10^{8}-10^{9} \mathrm{G}$ (e.g. Hartman et al. 2008) are capable to drive a portion of the accreting matter from the accretion disc to the magnetic polar caps of the compact object, heating its surface. The photons produced by this process are responsible for the pulsating X-ray emission (assuming that the NS magnetic and rotational axes are misaligned, as it is often the case). The timing analysis of the pul-

* pintore@iasf-milano.inaf.it sations can provide information about the strength of the NS magnetic fields and the inner radius of the accretion discs (e.g. Burderi et al. 2006, 2007; Burderi \& King 1998; Ghosh \& Becker 2008; Patruno et al. 2009; Wilkinson et al. 2011 or di Salvo et al. 2008 for a review). The latter can be also inferred from either the detection of disc emission or, indirectly, from broad emission features (the most diffuse feature is the K-shell transition iron line at $6.4-7.0 \mathrm{keV}$ ) in the source X-ray spectrum. These are likely produced by reflection off of hard photons from the surface of the accretion disc and are affected by special and general relativistic effects caused by the fast rotation of the accretion disc close to the strong gravitational field of the compact object (e.g. Fabian et al. 1989; Cackett et al. 2010; Papitto et al. 2009; Di Salvo et al. 2018, submitted, and references therein).

The spectral properties of AMXPs have been largely investigated (see e.g. Patruno \& Watts 2012; Burderi \& Di Salvo 2013; Campana \& Di Salvo 2018, and reference therein, for recent reviews) and showed that AMXPs are usually in hard states, characterized by the combination of a thermal soft component (temperatures $<2 \mathrm{keV}$ ), a dominating Comptonizating component with electrons in an optically thin hot plasma with temperatures of 20$50 \mathrm{keV}$, and in some cases of a third thermal continuum compo- 


\section{Pintore et al.}

nent likely produced by the NS surface (e.g. Gilfanov et al. 1998; Gierliński \& Poutanen 2005; Falanga et al. 2005; Patruno et al. 2009; Papitto et al. 2010; Papitto et al. 2013a). It is believed that the first soft component is the accretion disc, while the hard component may be arising form either a hot corona or the accretion columns or the boundary layer (e.g. Popham \& Sunyaev 2001).

In this work, we focus on the AMXP SAX J1748.9-2021, discovered by Beppo-SAX in 1998 during its first recorded outburst. The source is located in the globular cluster NGC 6440 (in 't Zand et al. 1999), at a distance of $\sim 8.5 \mathrm{kpc}$ and $0.6 \mathrm{kpc}$ above the Galactic plane (Martins et al. 1980; Ortolani et al. 1994; Kuulkers et al. 2003; Valenti et al. 2007). SAX J1748.9-2021 experienced further outbursts in 2001, 2005, 2010 and 2015 (in 't Zand et al. 1999; in't Zand et al. 2001; Verbunt et al. 2000; Markwardt \& Swank 2005; Patruno et al. 2009; Pintore et al. 2016; Sanna et al. 2016), with also a possible a posteriori associated outburst in 1971 (Markert et al. 1975). During quiescence, the companion star was detected (in't Zand et al. 2001) and its mass was estimated to be in the range 0.1$1 \mathrm{M}_{\odot}$ (Altamirano et al. 2008). The binary system has a period of $\sim 8.76 \mathrm{hr}$ and a projected semi-major axis of $\sim 0.4$ light-seconds (Altamirano et al. 2008; Patruno et al. 2009). SAX J1748.9-2021 showed intermittent pulsations at $\sim 442.361 \mathrm{~Hz}$ (Altamirano et al. 2008; Patruno et al. 2009; Sanna et al. 2016) and it is known to emit numerous type-I X-ray bursts (observed with RXTE and XMMNewton; e.g. Galloway et al. 2008; Pintore et al. 2016). During the 2015 outburst, the source was observed in a soft state, with average spectral properties consistent with two soft thermal components plus a cold thermal Comptonized component $(\sim 2 \mathrm{keV})$ and an additional hard X-ray emission described by a power-law ( $\Gamma \sim 2.3$, Pintore et al. 2016). These components were associated to the accretion disc, the NS surface and a thermal Comptonized emission coming out of an optically thick plasma region, respectively. The origin of the high energy tail was unclear, although a similar component has been detected in several other LMXBs during soft states (e.g. Di Salvo et al. 2000; Paizis et al. 2006; D'Aí et al. 2007; Tarana et al. 2007; Piraino et al. 2007).

In 2017, SAX J1748.9-2021 underwent its sixth outburst. The event was firstly detected by MAXI/GSC (Negoro et al. 2017) on September, 29th and then observed by Swift/XRT, INTEGRAL, XMM-Newton and NUSTAR (Bahramian et al. 2017; Harita et al. 2017; Di Gesu et al. 2017). Here we present the study of the 2017 broad-band spectral properties of SAX J1748.9-2021 using XMMNewton, NuSTAR, INTEGRAL and Swift/XRT observations.

\section{DATA REDUCTION}

We analysed XMM-Newton and NUSTAR DDT observations performed on October, 9th 2017 (Obs.ID. 0795712201) and October, 11th 2017 (Obs.ID. 90301320002), respectively. We also investigated all the Swift/XRT observations which monitored the source outburst (Figure 1-top), as well as all the available INTEGRAL observation taken between October, 7th and 13th.

XMM-Newton The XMM-Newton observation was taken in TIMING mode for a total exposure of $\sim 56 \mathrm{ks}$. We extracted EPICpn (Strüder et al. 2001) events only as the two EPIC-MOS cameras (Turner et al. 2001) were not pointing the source. We reduced the EPIC-pn data with SAS v15.0.0 (using the RDPHA corrections, e.g. Pintore et al. 2014), selecting single- and double-pixel events $($ PATTERN $\leqslant 4)$. We extracted source and background events from $\mathrm{RAWX}=[32: 44]$ and $\mathrm{RAWX}=[3: 5]$, respectively. We verified
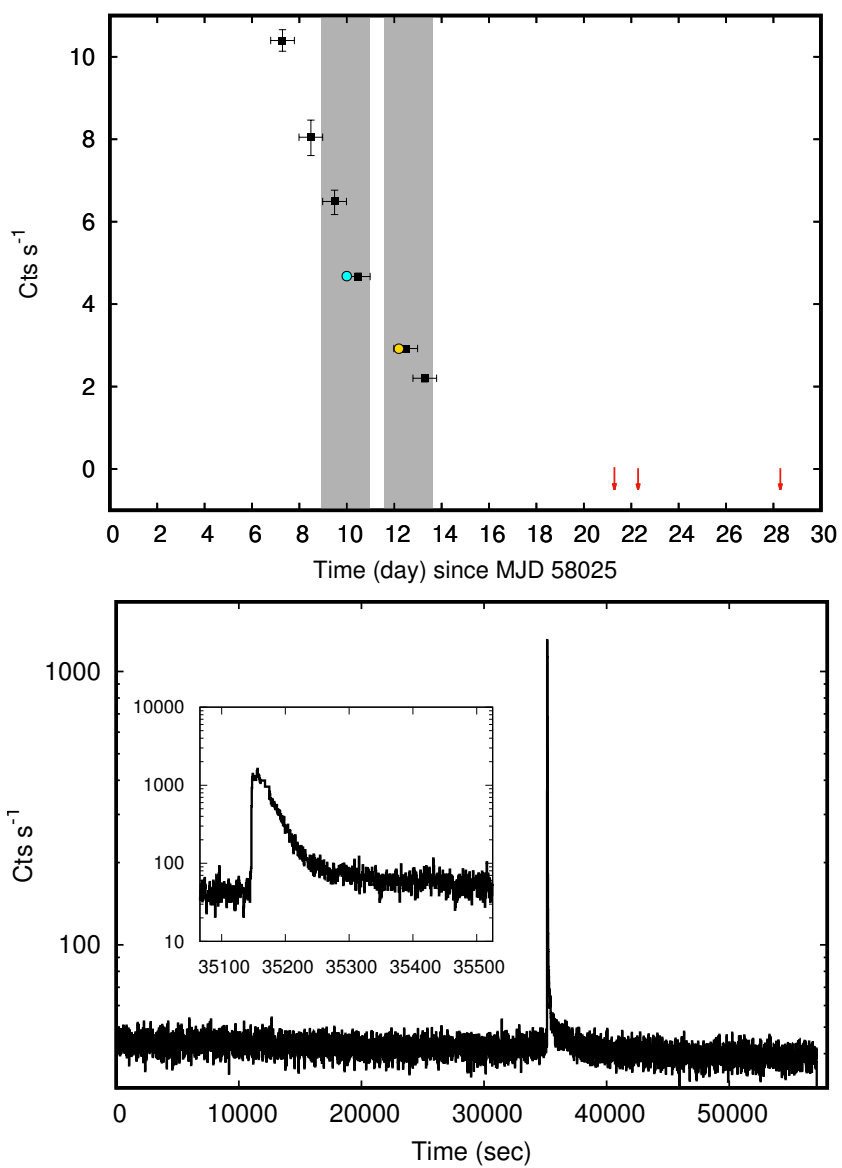

Figure 1. Top: background subtracted $0.3-10 \mathrm{keV}$ Swift-XRT lightcurve (starting from the beginning of the outburst as reported by MAXI), where red arrows are $3 \sigma$ upper limits; the XMM-Newton and NuSTAR observations have been taken at MJD 58035 (cyan circle) and 58037 (orange circle), respectively. INTEGRAL observations were taken in the epochs inside the grey boxes. Bottom: background subtracted 0.3-10 keV XMM/EPIC-pn lightcurve; a type-I burst (zoomed in the inset) happened at $\sim 35 \mathrm{ks}$ after the beginning of the observation.

that the background extracted in this region was not heavily contaminated by the source, comparing it with the background estimated from the EPIC-MOS data. During the observation, SAX J1748.9-2021 showed a type-I burst (Figure 1-bottom).

As the average EPIC-pn count rate was $\sim 30 \mathrm{cts} \mathrm{s}^{-1}$, it was well below the threshold for pile-up ${ }^{1}$. We extracted the average EPIC-pn spectrum and rebinned it with an oversample of 3 channels per energy resolution element using the specgroup task. The spectrum was analyzed in the range $1.3-10 \mathrm{keV}$, to limit the EPICpn calibration uncertainties at low energies when operated in TIMING mode.

The RGS spectra, first and second order, were extracted using the standard rgsproc task. We created an average spectrum merging RGS1 and RGS2 data (using the tool rgscombine which combines, separately, the first and second order spectra). We rebinned the final spectrum with at least 100 counts per bin and we fitted it in the range $0.6-2.0 \mathrm{keV}$

1 https://heasarc.gsfc.nasa.gov/docs/xmm/sas/USG/epicpileuptiming.html 
NuSTAR We followed the standard procedure based on NUSTARDAS (the NuSTAR Data Analysis Software v1.3.0) in the HEASOFT FTOOLS v6.23 to reduce the NuSTAR data and obtain cleaned event files. The total exposure time was $44 \mathrm{ks}$. We extracted source and background events from circular regions of radii 60" and 90", respectively. No type-I bursts were revealed during the observation. The FMPA and FMPB spectra were then grouped to have at least 100 counts per bin, respectively, and we fitted them in the range 3-70 keV.

Swift We used all the available 9 Swift/XRT observations collected between 2017, October 5 and 14, in both PC (the first and the last three observations of the monitoring) and WT mode (5 observations between 7-11 October). We followed standard procedures to reduce the data and extract spectra and lightcurves ${ }^{2}$. We grouped the spectra to have at least 25 counts per bin and we fitted them in the range 0.3-10 keV. During the first Swift observation (taken in PC mode), pile-up was not negligible and we corrected for its effect ${ }^{3}$ when we analyzed this data.

INTEGRAL We also analyzed the available INTEGRAL observations of the field around SAX J1748.9-2021 during the satellite revolutions 1871 and 1872 . These covered the time span from 2017 October 7 at 21:39 to October 9 at 22:47 (UTC) and from 2017 October 10 at 13:29 to October 12 at 14:49 (UTC), respectively. We analyzed all the publicly available INTEGRAL data and the data for which our group got data rights in AO14 by using version 10.2 of the Off-line Scientific Analysis software (OSA) distributed by the ISDC (Courvoisier et al. 2003). INTEGRAL observations are divided into "science windows" (SCWs), i.e. pointings with typical durations of $\sim 2-3 \mathrm{ks}$.

The source was within the JEM-X field of view (FoV; Lund et al. 2003) only for 6 SCWs during revolution 1872 and the total effective exposure time was of only $12.9 \mathrm{ks}$. As the source was located at the rim of the instrument FoV, the statistics of the data was too low to extract a meaningful spectrum. We used the JEM$\mathrm{X} 1$ and JEM-X2 lightcurves with a time resolution of $2 \mathrm{~s}$ to search for type-I X-ray bursts, but no significant detections were found.

We used for IBIS/ISGRI (Ubertini et al. 2003; Lebrun et al. 2003) all SCWs where the source was located within 12 degree from the center of the FoV. This provided a total exposure of 58.4 ks and $44.6 \mathrm{ks}$ in revolution 1871 and 1872 , respectively. We extracted two ISGRI spectra, one for each revolution. Both these spectra could be well fit with a simple power-law with photon index of $\sim 1.8$. As no spectral variability could be measured between the two revolutions, we also extracted the average ISGRI spectrum by combining all data available. Finally, we fit the ISGRI spectrum in the energy band $20 \mathrm{keV}-100 \mathrm{keV}$.

\section{RESULTS}

\subsection{Persistent emission}

\subsubsection{9th October}

We started our analysis with the XMM-Newton data, taken during the beginning of the decaying phase (9th October). Because the

2 http://www.swift.ac.uk/analysis/xrt/index.php

3 following the thread in http://www.swift.ac.uk/analysis/xrt/pileup.php first part of the ISGRI observations was taken on the same epoch and no significant spectral variability was found in these data, in order to have a broad-band spectrum we fitted simultaneously the EPIC-pn/RGS and ISGRI data.

On the basis of the spectral properties of the previous outburst, we initially adopted an absorbed NTHCOMP model (Zdziarski et al. 1996; Życki et al. 1999) in XSPEC (v. 12.8.2; Arnaud 1996). For the absorption, we used the TBABS model with the abundances of Wilms et al. (2000). We also added a multiplicative constant to take into account inter-calibration uncertainties between the different instruments and flux variations. We note that in the EPIC-pn spectrum there was a strong instrumental Au emission line (2.2 keV) and we fitted it with a gaussian in all the adopted models.

The TBABS $\times$ NTHCOMP fit did not provide acceptable results $\left(\chi^{2} /\right.$ dof $\left.=969.12 / 764\right)$, leaving several residuals especially in the band 1-10 keV. Therefore, we added a soft component (a DISKBB model; Mitsuda et al. 1984) that significantly improved the best-fit $\chi^{2}$ ( 899.87 for 763 dof). Its best-fit normalization, for a temperature of $0.76 \pm 0.04 \mathrm{keV}$ and for an inclination angle of $\sim 40^{\circ}$ (as found in Pintore et al. 2016), implies an implausible inner accretion disc radius of $\sim 1.7-3.1 \mathrm{~km}$. Hence, this was more likely modelling the emission from the NS surface and we changed the DISKBB with a BBODYRAD model that, as expected, did not change significantly the best fit and gave a temperature of $0.67 \pm 0.06 \mathrm{keV}$ and an emitting radius of $2.4 \pm 0.2 \mathrm{~km}$.

The sp residuals suggested to add also an emission line at the energy of the Iron K-shell transitions (6.4-6.9 keV), that we modelled with a gaussian. The Iron line has an energy of $6.5 \mathrm{keV}$, a width $(\sigma)$ of $0.1 \mathrm{keV}$ (although the uncertainties are large) and an equivalent width (EW) of $0.015 \mathrm{keV}$, which we suggest may be due to the reflection off of hard photons from the inner regions of the accretion disc (e.g. Fabian et al. 1989). We then tried to substitute the GAUSSIAN model with a DISKLINE model (Fabian et al. 1989). Adopting the more recent and constrained spectral estimates reported in Pintore et al. (2016), we fixed the inclination angle to $44^{\circ}$, the emissivity index to -2.7 and the outer disc radius at $10^{5}$ gravitational radii: as expected, this model does not change significantly the best fit, giving an energy line of $6.50 \pm 0.15 \mathrm{keV}$. The inner disc radius was poorly constrained $\left(\mathrm{R}_{i n}=43_{-43}^{+31} \mathrm{~km}\right)$ and no robust indication can be inferred. We tentatively adopted also selfconsistent reflection models as REFLIONX (Ross \& Fabian 2005) or RFXCONV (Kolehmainen et al. 2011) but they did not improve the fit and the spectral parameters could not be well constrained.

The final best-fit model (BBODYRAD + NTHCOMP + GAUSSIAN; $\chi^{2} /$ dof $\left.=875.85 / 774\right)$ provided the best-fit parameters reported in Table 1 and shown in Figure 2-left. We note that the $\mathrm{nH}$ is smaller than the one reported in Pintore et al. (2016). Such a discrepancy may be related to a possible variation of the local absorption during the latest outburst. Furthermore, we mention a caveat of our best-fit model as we found a degeneracy between the spectral parameters $\mathrm{kT}_{\text {seed }}$ and $\mathrm{kT}_{b b}$, as acceptable fits can be also obtained with the BBODYRAD temperature smaller than the seed photon temperature. In the case the two temperatures were linked, the best-fit converges towards a mean temperature of $0.53 \pm 0.2$ $\mathrm{keV}$ without a significant change in the other parameters but the statistical significance worsens.

Finally, we report that the averaged, unabsorbed $0.3-70 \mathrm{keV}$ flux of SAX J1748.9-2021 was $(3.6 \pm 0.03) \times 10^{-10} \mathrm{erg} \mathrm{cm}^{-2}$ $\mathrm{s}^{-1}$, corresponding to a luminosity of $\mathrm{L}_{x} \sim 3 \times 10^{36} \mathrm{erg} \mathrm{s}^{-1}$ (for a distance of $8.5 \mathrm{kpc}$ ), i.e. $\sim 1.6 \%$ of the Eddington limit. 

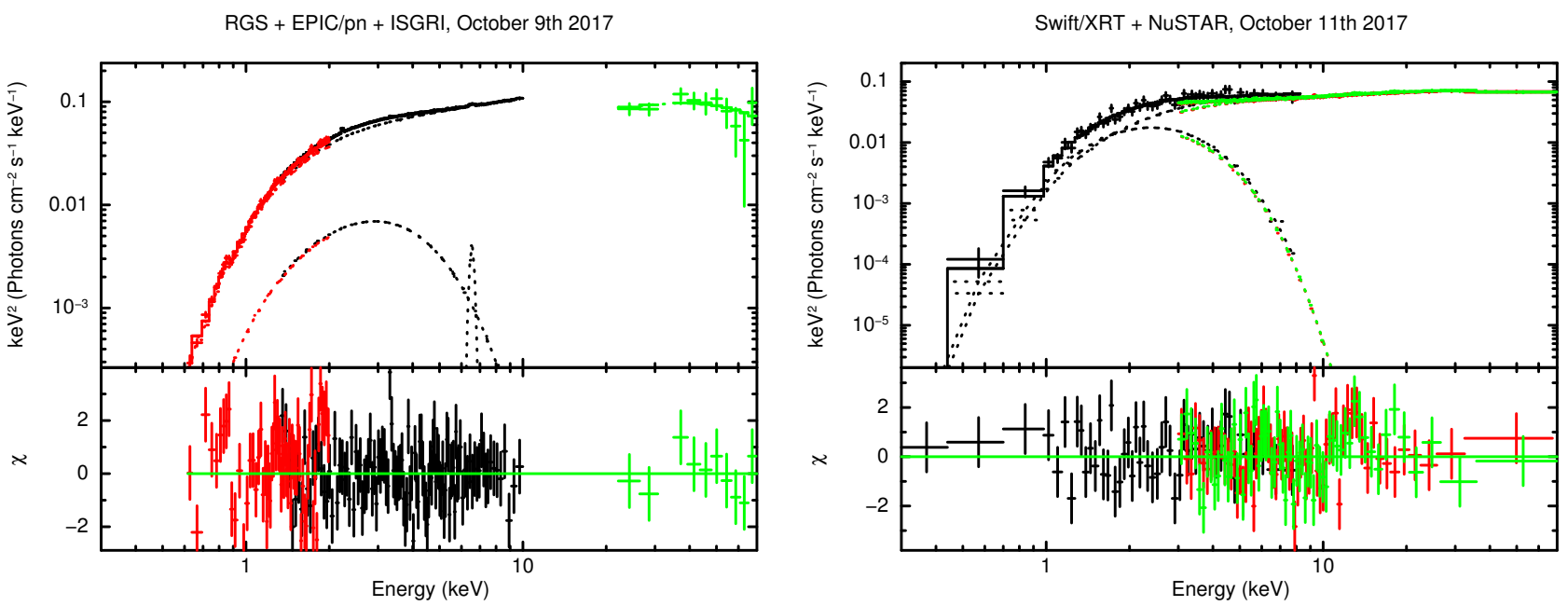

Figure 2. Left: unfolded $E^{2} f(E) X M M$-Newton/EPIC-pn (black), RGS (red) and ISGRI (green) spectra fitted with an absorbed BBODYRAD + NTHCOMP + GAUSSIAN model. Right: Swift/XRT (black), NuSTAR/FMPA and FMPB (red and green), fitted with an absorbed BBODYRAD + NTHCOMP model. Data have been rebinned for display purposes only.

Table 1. Best fit spectral parameters obtained with the BBODYRAD + NTHCOMP + GAUSSIAN model. Errors are at $90 \%$ for each parameter.

\begin{tabular}{llll}
\hline \multirow{2}{*}{ Model } & Component & $\begin{array}{l}\text { 9th Oct. } \\
\text { XMM+ISGRI }\end{array}$ & $\begin{array}{l}\text { 11th Oct. } \\
\text { XRT+NuSTAR }\end{array}$ \\
TBABS & $\mathrm{n}_{H}\left(10^{22} \mathrm{~cm}^{-2}\right)$ & $0.51_{-0.02}^{+0.02}$ & $0.62_{-0.12}^{+0.14}$ \\
BBODYRAD & $\mathrm{kT}_{b b}(\mathrm{keV})$ & $0.72_{-0.06}^{+0.04}$ & $0.54_{-0.04}^{+0.04}$ \\
& $\mathrm{norm}$ & $6.0_{-1.3}^{+1.1}$ & $29_{-9}^{+15}$ \\
& & & \\
NTHCOMP & $\Gamma(\mathrm{XMM})$ & $1.62_{-0.04}^{+0.03}$ & $1.79_{-0.01}^{+0.01}$ \\
& $\mathrm{kT}_{\text {seed }}(\mathrm{keV})$ & $0.43_{-0.03}^{+0.03}$ & $=\mathrm{kT}_{b b}$ \\
& $\mathrm{kT}_{e}(\mathrm{keV})$ & $17_{-4}^{+9}$ & $23_{-4}^{+3}$ \\
& $\mathrm{norm}\left(10^{-2}\right)$ & $1.98_{-0.07}^{+0.07}$ & $1.2_{-0.2}^{+0.2}$ \\
& & & \\
GAUSSIAN & Energy $(\mathrm{keV})$ & $6.54_{-0.07}^{+0.08}$ & $6.54^{*}$ \\
& $\sigma(\mathrm{keV})$ & $0.13_{-0.09}^{+0.13}$ & $0.13^{*}$ \\
& $\mathrm{norm}\left(10^{-5}\right)$ & $3.3_{-1.3}^{+1.7}$ & $<2.1 \times 10^{-5 * *}$ \\
& $\mathrm{EW} \mathrm{(keV)}$ & 0.015 & 0.004 \\
\hline & $\chi^{2} /$ dof & $875.85 / 774$ & $931.63 / 936$ \\
\hline & & &
\end{tabular}

* fixed to this value.

** $90 \%$ upper limit on the normalization.

\subsubsection{1th October}

We then analyzed the simultaneous Swift/XRT (Obs.ID: 00010341005) and NUSTAR spectra taken on the 11th of October. As done in the previous section, we described the broad-band continuum with a BBODYRAD + NTHCOMP model, obtaining a statistically significant fit $\left(\chi^{2} / d o f=931.24 / 935\right)$. However, as the seed photons temperature was quite unconstrained and possibly consistent with the BBODYRAD temperature, we linked them in the fit (best-fit reported in Table 1 and shown in Figure 2-right). We found a significant spectral variability in the photon index $(\Gamma \sim 1.8)$ and blackbody temperature $\left(\mathrm{kT}_{b b} \sim 0.55 \mathrm{keV}\right)$ with respect to the 9 th of October observation. We calculated that the averaged, unabsorbed 0.3-70 keV flux of SAX J1748.9-2021 was $(2.2 \pm 0.1) \times 10^{-10} \mathrm{erg} \mathrm{cm}^{-2} \mathrm{~s}^{-1}$, i.e. $\sim 40 \%$ lower than the flux measured with XMM-Newton+ISGRI. The flux on 11th October corresponds to a luminosity of $\mathrm{L}_{x} \sim 1.9 \times 10^{36} \mathrm{erg} \mathrm{s}^{-1}$ (for a distance of $8.5 \mathrm{kpc}$ ), i.e. about $1 \%$ of the Eddington limit.

Furthermore, we note that there is no evidence for an Iron emission line. Adding a gaussian line with energy and width fixed to those found in the previous observation, we estimated a $90 \%$ upper limit on the line normalization of $2.1 \times 10^{-5}$ photons $\mathrm{cm}^{-2}$ $\mathrm{s}^{-1}$ and on the equivalent width of $0.004 \mathrm{keV}$.

\subsubsection{5th-14th October}

Finally, we analyzed the average spectrum of each Swift/XRT observation during the period 5th-14th October. We fitted them, in the range $0.3-10 \mathrm{keV}$, with a TBABS $\times$ NTHCOMP model where we only fixed to $20 \mathrm{keV}$ the electron temperature (as the fits were insensitive to this parameter). We note that there was no statistical need to add a soft component in any observation. The single NTHCOMP model provided very good results in all cases $\left(\chi^{2} / \operatorname{dof} \sim 1\right)$, where we found that the inferred spectral parameters $\left(\mathrm{nH}, \Gamma\right.$ and $\left.\mathrm{kT}_{\text {seed }}\right)$ were all consistent within $2 \sigma$ with those reported in Table 1, implying no remarkable spectral variability.

\subsection{Type-I burst}

SAX J1748.9-2021 is a well known type-I X-ray burster and, during the 2017 outburst, we found at least two type-I bursts, one during the first Swift/XRT observation (at 19:32:14 UTC on October 5th) and one during the XMM-Newton observation (at 21:05:11 UTC on October 9th). In addition, MAXI/GSC detected a bright X-ray burst at 06:44 UTC on October 6th, which lasted for $10 \mathrm{~s}$ and consistent with the position of SAX J1748.9-2021 (Harita et al. 2017).

Because of the higher data quality, we focus on the burst observed in XMM-Newton. Modelling the burst lightcurve with an exponential function plus a constant, we found that the e-folding decay time is $25 \pm 0.5 \mathrm{~s}$, similar to those inferred from previous SAX J1748.9-2021 type-I bursts. 


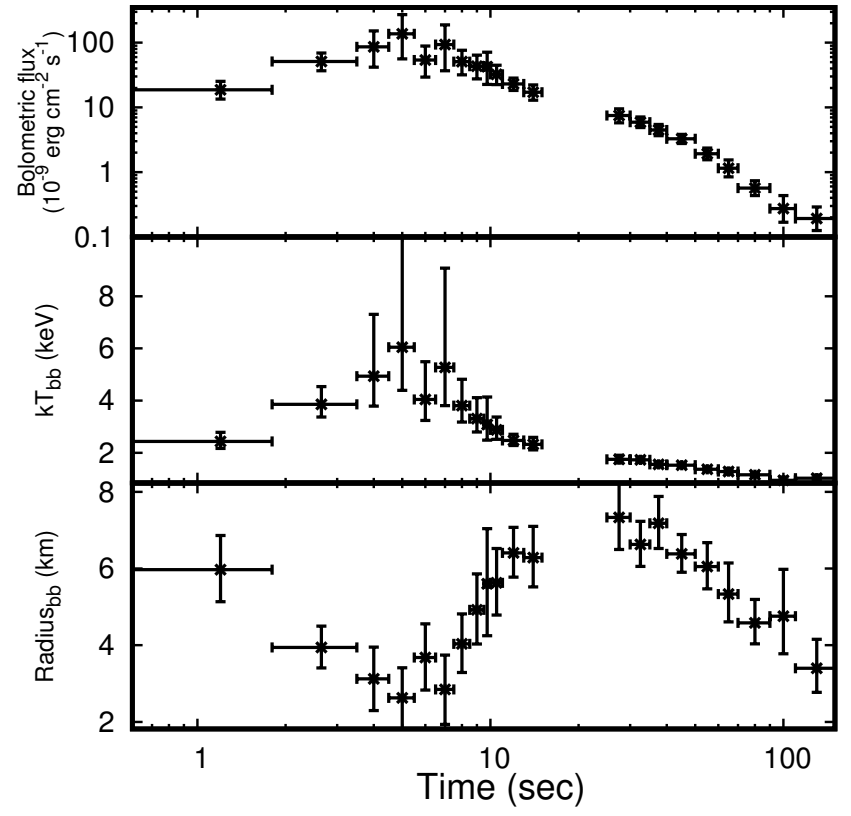

Figure 3. Bolometric flux, blackbody temperature and radius profiles of the type-I X-ray burst occurred during the XMM-Newton observation. The gap at $\sim 12 \mathrm{~s}-25 \mathrm{~s}$ is due to a temporary outage of the EPIC-pn instrument.

We then carried out a time-resolved spectroscopy, selecting a number of time-intervals from the beginning of the burst to $\sim 130$ s after it. For each interval, we extracted a spectrum (corrected for pile-up by excluding the central column RAWX $=37$ from the data) and we fitted it with an absorbed BBODYRAD model (fixing $\mathrm{nH}$ to $0.51 \times 10^{22} \mathrm{~cm}^{-2}$ ) in the range $1.0-10 \mathrm{keV}$, where we used as background the persistent emission pre-burst. The bolometric flux (estimated according to equation 3 in Galloway et al. 2008), the best-fit blackbody temperature, and the corresponding emitting radius are shown in Figure 3. The profile shows that the flux increased, peaked at a constant value for $\sim 10 \mathrm{~s}$, and then it decreased to the persistent emission level. A similar trend is observed for the temperature as well, which reached up to a maximum of $5 \mathrm{keV}$ (although with large uncertainties). Instead, the radius of the emitting regions has a more complex trend that resembles a photospheric radius expansion (PRE) event, which is not unusual for this source (Galloway et al. 2008; Güver \& Özel 2013). The mean bolometric flux registered at the peak (which lasted $\sim 10 \mathrm{~s}$ ) with XMM-Newton was $(50 \pm 4) \times 10^{-9} \mathrm{erg} \mathrm{cm}^{-2} \mathrm{~s}^{-1}$.

\section{DISCUSSION}

In this work we present the 2017 outburst of SAX J1748.9-2021 which lasted for $\sim 13$ days and with an exponential decay with efolding decay time of $\sim 4$ days. According to the MAXI lightcurve, SAX J1748.9-2021 reached the peak of the outburst between October, 4 and 5. Since the first Swift/XRT observation was taken on October, 5, we estimate that SAX J1748.9-2021 possibly reached at least an absorbed $0.3-70 \mathrm{keV}$ peak flux of $(7.0 \pm 0.7) \times 10^{-10}$ $\mathrm{erg} \mathrm{cm}^{-2} \mathrm{~s}^{-1}$, i.e. a factor of 2 higher than the flux measured during the latest $X M M$-Newton observation. This flux corresponds to a luminosity of $\mathrm{L}_{x} \sim 6.0 \times 10^{36} \mathrm{erg} \mathrm{s}^{-1}$ (for a distance of $8.5 \mathrm{kpc}$ ), hence about $4 \%$ of the Eddington luminosity.

Taking into account the marginal spectral variability during the decay, we found that the spectral properties of the 2017 outburst were characterized by a strong comptonized emission with electron and seed photon temperatures of $\sim 17-23 \mathrm{keV}$ and $0.4 \mathrm{keV}$, respectively, and photon index of 1.6-1.8. Such values correspond to an optical depth of $\tau \sim 3-5$ (see equation A1 in Zdziarski et al. 1996). We estimated that, for a Compton parameter $y \sim 3$, a bolometric flux of $3.4 \times 10^{-10} \mathrm{erg} \mathrm{s}^{-1}$ and a distance of $8.5 \mathrm{kpc}$, the radius of the region responsible for the seed photons is about 12 $\mathrm{km}$ (adopting the approach showed in the Discussion section of in 't Zand et al. 1999). It is therefore likely that the seed photons are produced in a region close to the NS, as the boundary layer or the NS surface, although we cannot rule-out contamination also from the accretion disc.

Furthermore, we found evidence of a significantly weaker blackbody component (although we remind here about the degeneracy between blackbody and seed photon temperature in the spectral fits discussed in Section 3.1.1) with a temperature of $\sim 0.7 \mathrm{keV}$ and emitting radius of a few $\mathrm{km}$, which origin is likely from the NS surface. This component carried $\sim 5 \%$ and $\sim 11 \%$ of the total source luminosity on October, 9 and October, 11, respectively.

On October, 9, it was also observed the presence of a relatively broad Iron line at $6.5 \mathrm{keV}$. We exclude that the broadening is either compatible with Compton down-scattering in a wind shell ejected at moderate relativistic velocities from the disc (Laurent \& Titarchuk 2007) or Compton processes in an accretion disc corona above the accretion disc (White \& Holt 1982; Kallman \& White 1989; Vrtilek et al. 1993). Indeed, should these processes responsible for the broadening, the corresponding electron temperature can be obtained from the relation $\Delta \epsilon / \epsilon=\left(4 k T_{e}-\epsilon\right) / m_{e} c^{2}$ (where $\Delta \epsilon$ is the line broadening and $\epsilon$ is its energy). However, we estimated an electron temperature of $\sim 4 \mathrm{keV}$ which is a factor of 4-5 lower than that found from our spectral analysis. Even if we consider the $90 \%$ upper uncertainties on the broadening and energy line (i.e. $0.26 \mathrm{keV}$ and $6.62 \mathrm{keV}$ ), we inferred an upper limit on the electron temperature of $\sim 7 \mathrm{keV}$, still well below our value. For this reason, we associate the line broadening to reflection processes from the surface of the accretion disc (see e.g. Fabian et al. 1989). Using the DISKLINE model, we could not constrain the dimension of the inner disc radius, and thus no definitive conclusion can be inferred on this parameter. Similar results are also obtained with self-consistent reflection models.

We remark that this is the sixth registered outburst of SAX J1748.9-2021, but its maximum peak luminosity is the weakest ever reported for this source to date (although Swift may underestimate the broadband source flux). In fact, all the other five events were significantly brighter, with the 1998 one being the shortest in duration and the lowest in peak luminosity (Altamirano et al. 2008). However the latter is still at least a factor of 3 higher than the peak luminosity of the 2017 outburst. For the 1998 outburst, using BeppoSAX data, in 't Zand et al. (1999) found that the $0.1-100 \mathrm{keV}$ spectrum of SAX J1748.9-2021 was consistent with a single thermal Comptonization model with electron temperature of $15 \mathrm{keV}$, optical depth in the range 3-6 and seed photon temperature of 0.6 $\mathrm{keV}$ (coming from a region of radius of $\sim 13 \mathrm{~km}$ ). This indicates that the source was in a hard state. Based on our analysis, we suggest that SAX J1748.9-2021 was again in a hard state during the 2017 outburst (and possibly all along the decay as the Swift/XRT monitoring did not show any clear evidence of spectral variability) making the two outbursts very similar. Pulsations have been mainly observed during the hard states of SAX J1748.9-2021 and we confirm a pulse detection, that we will report in greater detail elsewhere (Sanna et al. in prep.).

The last outburst was hence clearly different from the 2015 
one, when the source reached $\sim 25 \%$ of the Eddington luminosity, and its spectral properties were consistent with a marked soft state (Pintore et al. 2016). In particular, it was characterized by the combination of four spectral continuum components, representing the disc emission, the NS surface, a comptonized region (possibly the boundary layer) and an additional non-thermal emission (described with a hard powerlaw), carrying $\sim 20 \%, 25 \%, 51 \%$ and $3 \%$ of the total luminosity, respectively. In addition, the NS surface emission in 2015 had a temperature of $\sim 1.1 \mathrm{keV}$, about a factor of 2 higher than the temperature estimated in this work. In 2017, we also did not find any evidence of a hard powerlaw tail at high energy, confirming the hard state of the source, as such a component is generally seen only during soft states of LMXBs (e.g Di Salvo et al. 2000, 2001; D'Amico et al. 2001; Iaria et al. 2004; Di Salvo et al. 2006; Paizis et al. 2006; D'Aí et al. 2007; Tarana et al. 2007; Piraino et al. 2007).

At least three type-I X-ray bursts were observed from SAX J1748.9-2021 with MAXI, Swift/XRT and XMM-Newton in its 2017 outburst unlike the 1998 outburst where none of them were found. According to the linear relation between pre-burst count rate and elapsed time reported in Pintore et al. (2016) for the 2015 outburst, we predict that the expected recurrence time of the type-I bursts during the 2017 outburst would have been at least one every $\sim 8-9 \mathrm{ks}$, much shorter than the observed recurrence time. During the burst, the measured NS surface temperature increases up to $\sim 5 \mathrm{keV}$ (although the uncertainties are quite large). On the other hand, the radius of the emitting area presents a more complex trend: in fact, it is $\sim 6 \mathrm{~km}$ at the beginning of the burst and it decreases below $\sim 4 \mathrm{~km}$ during the burst rising phase; at the maximum flux, the radius increased again to $\sim 6 \mathrm{~km}$, remaining constant for at least 60 seconds. This behaviour is similar to the $r a-$ dius expansion process, i.e. the response of the NS outermost layers to a super-Eddington burst flux (see e.g. Kuulkers et al. 2003). In particular, the NS photosphere increases and expands and then contracts back to the NS surface. It was found that during the expansion/contraction phase the flux remains nearly constant to the Eddington limit, converting any excess into kinetic energy of the outflow (e.g. Kato 1983; Ebisuzaki et al. 1983; Paczynski \& Proszynski 1986). We note that radius expansions are not unusual for SAX J1748.9-2021 (e.g. Güver et al. 2012) although in the 2015 outburst they were not observed in any of the detected type-I X-ray bursts. Assuming that the mean flux at the peak of the burst reported here $\left(5.0 \times 10^{-8} \mathrm{erg} \mathrm{cm}^{-2} \mathrm{~s}^{-1}\right)$ reached the Eddington limit and using the Eddington luminosity empirically obtained by Kuulkers et al. (2003) $\left(3.8 \times 10^{38} \mathrm{erg} \mathrm{s}^{-1}\right)$, we estimated a source distance of $8.0 \pm 0.4 \mathrm{kpc}$ and $5.2 \pm 0.4 \mathrm{kpc}$ (error at $1 \sigma$ ) for a pure helium $(X=0)$ or a solar composition burst $(X=0.7)$, respectively, which are well consistent with the previous distance estimates (Galloway et al. 2008).

\section{ACKNOWLEDGEMENTS}

We thank both Dr. F. Harrison and Dr. N. Schartel, who made these ToO observations possible using their Director Discretionary Time, and the XMM-Newton and the NUSTAR team who performed and supported these observations. We used observations obtained with XMM-Newton, an ESA science mission with instruments and contributions directly funded by ESA Member States and NASA. We acknowledge financial contribution from the agreement ASI-INAF I/037/12/0. We acknowledges support from the HERMES Project, financed by the Italian Space Agency (ASI) Agreement n. 2016/13
U.O, as well as fruitful discussion with the international team on "The disk-magnetosphere interaction around transitional millisecond pulsars at the International Space Science Institute, Bern".

\section{REFERENCES}

Altamirano D., Casella P., Patruno A., Wijnands R., van der Klis M., 2008, ApJ, 674, L45

Archibald A. M., Stairs I. H., Ransom S. M., Kaspi V. M., et al. 2009, Science, 324, 1411

Arnaud K. A., 1996, in Jacoby, G. H. and Barnes, J., eds., Astronomical Data Analysis Software and Systems V. Vol. 101 of ASP Conf. Ser., San Francisco CA, XSPEC: The First Ten Years. p. 17

Bahramian A., Kennea J. A., Altamirano D., Beri A., Heinke C. O., Sivakoff G. R., Tetarenko A. J., Wijnands R., Degenaar N., 2017, The Astronomer's Telegram, 10826

Burderi L., Di Salvo T., 2013, Mem. Soc. Astron. Italiana, 84, 117

Burderi L., Di Salvo T., Lavagetto G., Menna M. T. e. a., 2007, ApJ, 657, 961

Burderi L., Di Salvo T., Menna M. T., Riggio A., Papitto A., 2006, ApJ, 653, L133

Burderi L., King A. R., 1998, ApJ, 505, L135

Cackett E. M., Miller J. M., Ballantyne D. R., Barret D., Bhattacharyya S., Boutelier M., Miller M. C., Strohmayer T. E., Wijnands R., 2010, ApJ, 720, 205

Campana S., Di Salvo T., 2018, Chapter of the book "Physics and Astrophysics of Neutron Stars", NewCompStar COST Action, 1304

Courvoisier T. J.-L., Walter R., Beckmann V., Dean A. J., Dubath P. e. a., 2003, A\&A, 411, L53

D’Aí A., Życki P., Di Salvo T., Iaria R., Lavagetto G., Robba N. R., 2007, ApJ, 667, 411

D'Amico F., Heindl W. A., Rothschild R. E., Gruber D. E., 2001, ApJ, 547, L147

Di Gesu L., Bozzo E., Kuulkers E., Bazzano A. e. a., 2017, The Astronomer's Telegram, 10832

di Salvo T., Burderi L., Riggio A., Papitto A., Menna M. T., 2008, in Yuan Y.-F., Li X.-D., Lai D., eds, Astrophysics of Compact Objects Vol. 968 of American Institute of Physics Conference Series, Timing of accreting millisecond pulsars. pp 233-237

Di Salvo T., Goldoni P., Stella L., van der Klis M., Bazzano A., Burderi L., Farinelli R., Frontera F., Israel G. L., Méndez M., Mirabel I. F., Robba N. R., Sizun P., Ubertini P., Lewin W. H. G., 2006, ApJ, 649, L91

Di Salvo T., Robba N. R., Iaria R., Stella L., Burderi L., Israel G. L., 2001, ApJ, 554, 49

Di Salvo T., Stella L., Robba N. R., van der Klis M., Burderi L., Israel G. L., Homan J., Campana S., Frontera F., Parmar A. N., 2000, ApJ, 544, L119

Ebisuzaki T., Hanawa T., Sugimoto D., 1983, PASJ, 35, 17

Fabian A. C., Rees M. J., Stella L., White N. E., 1989, MNRAS, 238, 729

Falanga M., Kuiper L., Poutanen J., Bonning E. W., Hermsen W., di Salvo T., Goldoni P., Goldwurm A., Shaw S. E., Stella L., 2005, A\&A, 444, 15

Galloway D. K., Muno M. P., Hartman J. M., Psaltis D., Chakrabarty D., 2008, ApJS, 179, 360

Ghosh P., Becker W., 2008, Physics Today

Gierliński M., Poutanen J., 2005, MNRAS, 359, 1261

Gilfanov M., Revnivtsev M., Sunyaev R., Churazov E., 1998, A\&A, 338, L83

Güver T., Özel F., 2013, ApJ, 765, L1

Güver T., Özel F., Psaltis D., 2012, ApJ, 747, 77

Harita S., Sugita S., Sakamaki A., Negoro H. e. a., 2017, The Astronomer's Telegram, 10827

Hartman J. M., Patruno A., Chakrabarty D., Kaplan D. L., Markwardt C. B., Morgan E. H., Ray P. S., van der Klis M., Wijnands R., 2008, ApJ, 675, 1468

Iaria R., Di Salvo T., Robba N. R., Burderi L., Stella L., Frontera F., van der Klis M., 2004, ApJ, 600, 358 
in 't Zand J. J. M., Verbunt F., Strohmayer T. E., Bazzano A., Cocchi M., Heise J., van Kerkwijk M. H., Muller J. M., Natalucci L., Smith M. J. S., Ubertini P., 1999, A\&A, 345, 100

in't Zand J. J. M., van Kerkwijk M. H., Pooley D., Verbunt F., Wijnands R., Lewin W. H. G., 2001, ApJ, 563, L41

Kallman T., White N. E., 1989, ApJ, 341, 955

Kato M., 1983, PASJ, 35, 33

Kolehmainen M., Done C., Díaz Trigo M., 2011, MNRAS, 416, 311

Kuulkers E., den Hartog P. R., in't Zand J. J. M., Verbunt F. W. M., Harris W. E., Cocchi M., 2003, A\&A, 399, 663

Laurent P., Titarchuk L., 2007, ApJ, 656, 1056

Lebrun F., Leray J. P., Lavocat P., Crétolle J., Arquès M., Blondel C. e. a., 2003, A\&A, 411, L141

Lund N., Budtz-Jørgensen C., Westergaard N. J., Brandt S., Rasmussen I. L. e. a., 2003, A\&A, 411, L231

Markert T. H., Backman D. E., Canizares C. R., Clark G. W., Levine A. M., 1975, Nature, 257, 32

Markwardt C. B., Swank J. H., 2005, The Astronomer's Telegram, 495, 1

Martins D. H., Harvel C. A., Miller D. H., 1980, AJ, 85, 521

Mitsuda K., Inoue H., Koyama K., Makishima K., Matsuoka M., Ogawara Y., Suzuki K., Tanaka Y., Shibazaki N., Hirano T., 1984, PASJ, 36, 741

Negoro H., Ueno S., Tomida H., Ishikawa M. e. a., 2017, The Astronomer's Telegram, 10821

Ortolani S., Barbuy B., Bica E., 1994, A\&AS, 108, 653

Paczynski B., Proszynski M., 1986, ApJ, 302, 519

Paizis A., Farinelli R., Titarchuk L., Courvoisier T. J.-L., Bazzano A., Beckmann V., Frontera F., Goldoni P., Kuulkers E., Mereghetti S., Rodriguez J., Vilhu O., 2006, A\&A, 459, 187

Papitto A., D’Aì A., Di Salvo T., Egron E., Bozzo E., Burderi L., Iaria R., Riggio A., Menna M. T., 2013a, MNRAS, 429, 3411

Papitto A., Di Salvo T., D’Aì A., Iaria R., Burderi L., Riggio A., Menna M. T., Robba N. R., 2009, A\&A, 493, L39

Papitto A., Ferrigno C., Bozzo E., Rea N., Pavan L., Burderi L., et al. 2013b, Nature, 501, 517

Papitto A., Riggio A., di Salvo T., Burderi L., D’Aì A., Iaria R., Bozzo E., Menna M. T., 2010, MNRAS, 407, 2575

Patruno A., Altamirano D., Hessels J. W. T., Casella P., Wijnands R., van der Klis M., 2009, ApJ, 690, 1856

Patruno A., Haskell B., Andersson N., 2017, ApJ, 850, 106

Patruno A., Rea N., Altamirano D., Linares M., Wijnands R., van der Klis M., 2009, MNRAS, 396, L51

Patruno A., Watts A. L., 2012, ArXiv e-prints

Pintore F., Sanna A., Di Salvo T., Del Santo M., Riggio A., D'Aì A., Burderi L., Scarano F., Iaria R., 2016, MNRAS, 457, 2988

Pintore F., Sanna A., Di Salvo T., Guainazzi M., D'Aì A., Riggio A., Burderi L., Iaria R., Robba N. R., 2014, MNRAS, 445, 3745

Piraino S., Santangelo A., di Salvo T., Kaaret P., Horns D., Iaria R., Burderi L., 2007, A\&A, 471, L17

Popham R., Sunyaev R., 2001, ApJ, 547, 355

Ross R. R., Fabian A. C., 2005, MNRAS, 358, 211

Sanna A., Bahramian A., Bozzo E., Heinke C., et al. 2018, A\&A, 610, L2

Sanna A., Burderi L., Riggio A., Pintore F., Di Salvo T., Gambino A. F., Iaria R., Matranga M., Scarano F., 2016, MNRAS, 459, 1340

Strohmayer T. E., Ray P. S., Gendreau K. C., Bult P. M., Guillot S., Mahmoodifar S., Jaisawal G. K., Arzoumanian Z., Altamirano D., Bogdanov S., Chakrabarty D., Enoto T., Markwardt C. B., Ozel F., Ransom S. M., 2018, The Astronomer's Telegram, 11507

Strüder L., et al., 2001, A\&A, 365, L18

Tarana A., Bazzano A., Ubertini P., Zdziarski A. A., 2007, ApJ, 654, 494

Turner M. J. L., et al., 2001, A\&A, 365, L27

Ubertini P., Lebrun F., Di Cocco G., Bazzano A., Bird A. J., Broenstad K. e. a., 2003, A\&A, 411, L131

Valenti E., Ferraro F. R., Origlia L., 2007, AJ, 133, 1287

Verbunt F., van Kerkwijk M. H., in't Zand J. J. M., Heise J., 2000, A\&A, 359,960

Vrtilek S. D., Soker N., Raymond J. C., 1993, ApJ, 404, 696

White N. E., Holt S. S., 1982, ApJ, 257, 318

Wilkinson T., Patruno A., Watts A., Uttley P., 2011, MNRAS, 410, 1513
Wilms J., Allen A., McCray R., 2000, ApJ, 542, 914

Zdziarski A. A., Johnson W. N., Magdziarz P., 1996, MNRAS, 283, 193

Życki P. T., Done C., Smith D. A., 1999, MNRAS, 309, 561 